\title{
Diversity in the organisation and lignification of tension wood fibre walls - A review
}

\author{
Barbara Ghislain* and Bruno Clair \\ CNRS, UMR EcoFoG, AgroParisTech, Cirad, INRA, Université des Antilles, \\ Université de Guyane, 97310 Kourou, France \\ *Corresponding author; e-mail: barbara.ghislain@gmail.com
}

\begin{abstract}
Tension wood, a tissue developed by angiosperm trees to actively recover their verticality, has long been defined by the presence of an unlignified cellulosic inner layer in the cell wall of fibres, called the G-layer. Although it was known that some species have no G-layer, the definition was appropriate since it enabled easy detection of tension wood zones using various staining techniques for either cellulose or lignin. For several years now, irrespective of its anatomical structure, tension wood has been defined by its high mechanical internal tensile stress. This definition enables screening of the diversity of cell walls in tension wood fibres. Recent results obtained in tropical species with tension wood with a delay in the lignification of the G-layer opened our eyes to the effective presence of large amounts of lignin in the G-layer of some species. This led us to review older literature mentioning the presence of lignin deposits in the G-layer and give them credit. Advances in the knowledge of tension wood fibres allow us to reconsider some previous classifications of the diversity in the organisation of the fibre walls of the tension wood.
\end{abstract}

Keywords: Tension wood, fibres, G-layer, $S_{1}+S_{2}+S_{3}+G$, lignification, multilayered tension wood fibre walls, diversity.

[In the online version of this paper Figure 2 is reproduced in colour.]

\section{INTRODUCTION}

\section{Definition of tension wood}

\section{Historical definition of tension wood}

Trees are fascinating slender structures as they can combine reasonable trunk diameter with impressive height. This performance is possible thanks to the ability of the tree to form pre-stressed material (Archer 1987). This internal stress is the result of a process which occurs during cell maturation and enables control of tree posture (Moulia $\&$ Fournier 2009). When the trunk or branches need reorientation, asymmetric stress distribution is induced thanks to a special kind of wood called reaction wood. More precisely, compression wood, which generates compressive stress on the lower part of the tilted stem in gymnosperms, can be distinguished from tension wood, which generates tensile stress on the upper part of the tilted stem in angiosperm trees. This tissue allows the tree to continuously adjust its position to the multiple disturbances that occur throughout its life. 
Studies on compression wood were exhaustively summarized by Timell (1986) and recently updated by Gardiner et al. (2014a). This most recent book, "The Biology of Reaction Wood" (Gardiner et al. 2014a), also dedicated a large section to tension wood in which the structure, function, and properties of tension wood are described in detail from the scale of the tree down to that of macromolecules. Ruelle (2014) reported the structure of tension wood from macroscopic scale to nanometric scales. In this paper we thus focus on the organisation and composition of the tension wood cell wall, with particular attention to its diversity in angiosperms (excepting the few angiosperms which form compression wood), and reinterpret old data in the light of recent results.

Today, tension wood is defined by measuring the mechanical strain resulting from the release of stress around the tree circumference, independently of the anatomy of the wood which is formed (Fournier et al. 2014). This mechanical definition finally allows the description of the anatomical diversity of tension wood.

However, in the literature only a few studies included strain measurements on the living tree. Most studies identified tension wood thanks to the tree shape: tilted trees with the trunk recovering verticality or branches where the mechanical effort to maintain branch angle is obvious. Additionally several studies were performed on young trees artificially tilted to ensure the production of tension wood. Unfortunately, we found no literature comparing the tension wood anatomy caused by different mechanical stimuli (artificial or natural) or occurring in trunks or branches. One can however suspect that tension wood cell wall type does not depend on the stimulus, based on the numerous studies on Populus sp. where the tension wood cell wall is always reported with a similar structure regardless of the type of stimulus or axis studied.

From an anatomical point of view, tension wood has often been defined by the presence of an internal gelatinous layer in the fibres (IAWA 1964). The so-called gelatinous layer, or G-layer, was discovered in the $19^{\text {th }}$ century by Hartig (after Sanio 1860a cited in Potter 1904). Because of its high cellulose content and its anatomically swollen, detached and jelly-like aspect, the layer was originally called cellulosic layer, mucilaginous layer, cartilaginous layer, or gelatinous layer (Sanio 1860a, 1860b, 1863; Potter 1904; Metzger 1908). Ultimately, gelatinous layer or G-layer became the official name. Its detached and jelly-like aspect was later reported to be a border artefact occurring during sectioning due to the high tensile stress in the G-layer compared to the $\mathrm{S}_{2}$ layer and the weak transverse stiffness of the G-layer (Clair et al. 2005a \& b). Unless the wood specimen is embedded in resin and the first $100 \mu \mathrm{m}$ are trimmed, the detachment and swelling of the G-layer should be observable. Therefore, on 20-50 $\mu \mathrm{m}$ unembedded sections, the G-layer is usually swollen and detached from the other layers. Evidence for the presence of a gel in the G-layer was provided much later thanks to the characterisation of its mesoporous texture, with pores ranging from 2 to $50 \mathrm{~nm}$ (Clair et al.2008).

\section{Poplar as a model for studies of tension wood with a G-layer}

Although early on it was known that some species lack G-layers (Onaka 1949), most studies were carried out on tension wood with G-layers, but instead of studying the G-layer in different species, the literature converged on poplar as a model tree (the 
Appendix illustrates the increasing focus on poplar in tension wood studies). Poplar was chosen for its many advantages: it is a fast-growing species which makes it possible to obtain results rapidly; it is a wood of commercial interest in which tension wood is responsible for wood defects including a woolly surface; tension wood is anatomically easy to locate thanks to its thick G-layer; and more recently it has become possible to choose different well-characterised genotypes thanks to the description of the poplar genome (Pilate et al. 2004b; Tuskan et al. 2004).

The G-layer is an internal layer in the cell wall of fibres, which replaces the $\mathrm{S}_{3}$ and part of the $S_{2}$ layer in poplar (Wardrop \& Dadswell 1955). It was historically described to be mostly, or even only, composed of cellulose. More recently, several authors demonstrated the presence of other polysaccharides and proteins (Lafarguette et al. 2004; Andersson-Gunnerås et al. 2006; Nishikubo et al. 2007; Bowling \& Vaughn 2008, whose study was on Celtis and Liquidambar; Kim \& Daniel 2012). The cellulose microfibrils of the G-layer are highly crystalline and are almost aligned with the axis of the fibre (Fujita et al. 1974; Chaffey 2000; Daniel et al. 2006). In the process of G-layer deposition during cell wall maturation, a strong tensile stress occurs in its cellulose microfibrils (Clair et al. 2011) associated with the swelling of the mesopores (Chang et al. 2015). And indeed, the thicker the G-layer, the greater the maturation stress (Fang et al. 2007).

The absence of lignin in the G-layer of poplar tension wood has been demonstrated in several studies (Norberg \& Meier 1966; Pilate et al. 2004a; Yoshinaga et al. 2012), whereas other studies reported the presence of lignin (see below). However, the most frequently cited paper providing evidence for lignin (Joseleau et al. 2004) only qualifies its presence as 'traces'. As a result, the lack of lignification has become a strong criterion to define the G-layer of poplar. As this species has been extensively studied, the criterion is used by extension to define tension wood in many other hardwood species. Tension wood thereby became easy to locate thanks to different types of staining which made it possible to distinguish the cellulose G-layer from the other lignified layers. As lignification does not occur in the G-layer, when tension wood did show slight lignin staining, the sample was often discarded (Gartner et al. 2003). At that time, stains such as safranin/alcian blue were considered to be insufficiently specific and the traces of safranin in the G-layer were often considered as a staining problem. Indeed, observations with UV light (which highlighted the lignin in unstained samples thanks to their auto-fluorescence) or the use of Wiesner or Mäule tests are known to be more specific. However, comparison of safranin/alcian blue with observations using UV light demonstrated the reliability of such double staining (Roussel \& Clair 2015; personal observations) as long as the sample is sapwood and does not contain heartwood extractives. Therefore, in a number of observations reported in the literature, safranin staining of the G-layer may not have been a staining problem but, in contrast, may have revealed traces of lignin in the G-layer.

It should be noted that G-layer fibres can also be found in straight trees, scarcely dispersed, or as tangential bands around the circumference of the trunk (Badia et al. 2006). These observations reveal that short reactions were needed to maintain the tree upright. The few G-fibres on one side enabled vertical recovery at the time of their 
development. Then, later in tree growth, an imbalance in another direction made it necessary to produce G-fibres on the other side of the stem, etc.

\section{Different organisation of the cell wall layers in gelatinous fibres}

Three types of organisation of the cell wall of G-fibres were proposed by Wardrop and Dadswell (1955): 1) $S_{1}+S_{2}+G$; 2) $S_{1}+G$; and 3) $S_{1}+S_{2}+S_{3}+G$. They found that tension wood of Eucalyptus gigantea had $\mathrm{S}_{1}+\mathrm{S}_{2}+\mathrm{G}$ in latewood and $\mathrm{S}_{1}+\mathrm{G}$ in earlywood. $\mathrm{S}_{1}+\mathrm{S}_{2}+\mathrm{G}$ is the most common type and occurs in 10 out of the 12 species studied by Saiki and Ono (1971). Only Cercidiphylum japonicum presented two types, $\mathrm{S}_{1}+\mathrm{G}$ and $\mathrm{S}_{1}+\mathrm{S}_{2}+\mathrm{G}$, in the same portion of tension wood. The third type is quite rare. The only reports we found in the literature were by Wardrop and Dadswell (1955), Saiki and Ono (1971) and Araki et al. (1983). Unfortunately, in the first paper, Wardrop and Dadswell (1955) did not mention the position of the sampling, i.e. they did not specify if sampling was done during the process of maturation or in a transition zone between normal wood and tension wood. Furthermore, it cannot be checked, since the observation was made on an unidentified Flacourtiaceae (currently split into two families Achariaceae and Salicaceae sensu lato) and, as we now know, many Flacourtiaceae newly classified as Salicaceae sensu lato are able to form multi-layered fibre walls in their tension wood (Ghislain et al. 2016). The illustration of Wardrop and Dadswell (1955) does not allow us to confirm the presence of an $\mathrm{S}_{3}$ layer or a multi-layered G-layer, since Ruelle et al . (2007c) described the thin lignified layers in multi-layered tension wood as similar to the $\mathrm{S}_{3}$ layer. Saiki and Ono (1971) also mentioned the third type of organisation $\left(\mathrm{S}_{1}+\mathrm{S}_{2}+\mathrm{S}_{3}+\mathrm{G}\right)$ in thin sections of resin-embedded samples of Celtis sinensis for which methacrylate was removed before Pt-Pd shadowing, but were unable to confirm it in thin sections of Epon-embedded samples stained with potassium permanganate. Finally, Araki et al. (1983) also found the third type, but only in the transition zone between normal wood and tension wood. This is in agreement with the results of Abedini et al. (2015) on poplar, in which the authors reported the establishment of a G-layer on an $\mathrm{S}_{2}$ layer formed before the tilting of the tree. It could therefore be hypothesised that in the study by Araki et al. (1983) the $\mathrm{S}_{3}$ layer was formed just before tilting and the formation of a G-layer, thus explaining the presence of the organisation $\mathrm{S}_{1}+\mathrm{S}_{2}+\mathrm{S}_{3}+\mathrm{G}$ in the transition zone only.

It would thus seem that the type $\mathrm{S}_{1}+\mathrm{S}_{2}+\mathrm{S}_{3}+\mathrm{G}$, can only be observed in the transition zone from normal wood to tension wood, and that only two types of cell wall organisation can occur in tension wood: $S_{1}+S_{2}+G$ and $S_{1}+G$. Further investigations are required to clarify this issue.

\section{Tension wood classified as with or without a G-layer}

Onaka (1949) was the first to carry out a large study on both compression and tension wood and investigated the latter in 219 species belonging to 84 genera of dicotyledons. His classification, although very interesting, is sometimes a little confusing due to the use of the growth eccentricity as a criterion for classification (Fig. 1).

At first, when an eccentricity was present on the upper side, it was classified as being present either both in the wood and in the bark or only in the wood, even if the 


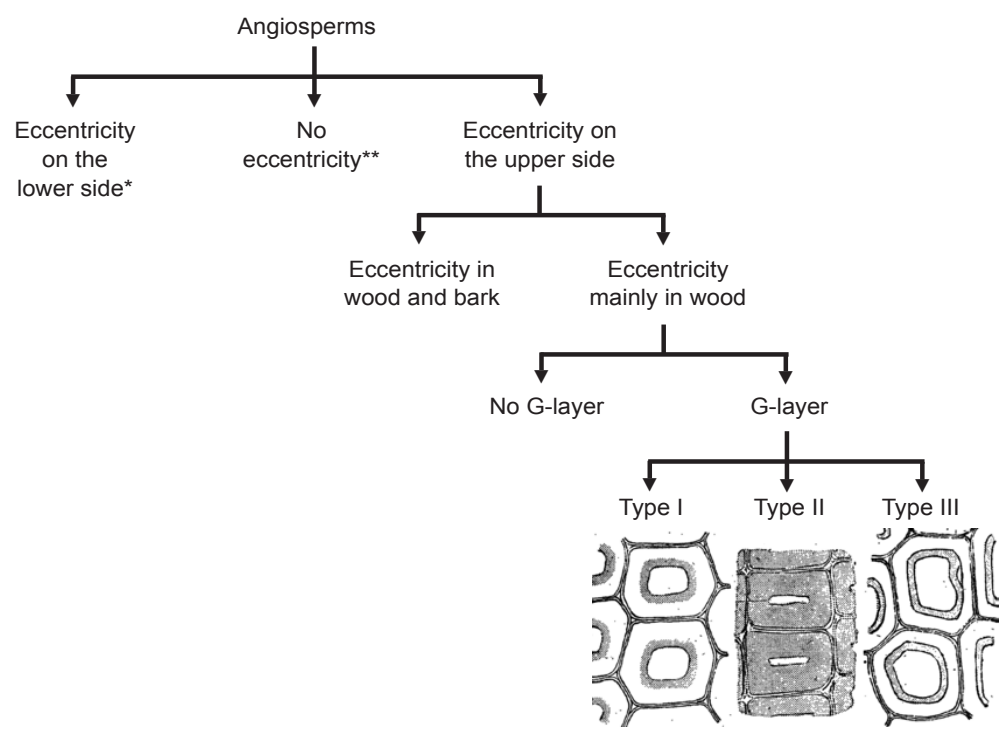

Figure 1. Schematic diagram of the classification proposed by Onaka (1949). *: Class containing only Gardenia and Buxus. **: Class represented by shrubs or vines and two trees, Paulownia tomentosa and Catalpa ovata. Pictures of the cells are the original drawings by Onaka (1949), the unlignified G-layers are in gray.

bark developed more on the upper side. The author then divided the latter according to the presence or absence of the G-layer. Finally, when the G-layer was present, the author defined three types (I, II and III). These three types are not easily distinguishable but Wardrop and Dadswell (1955) presumed the types corresponded to the $\mathrm{S}_{1}+\mathrm{G}$, $\mathrm{S}_{1}+\mathrm{S}_{2}+\mathrm{G}$ and $\mathrm{S}_{1}+\mathrm{S}_{2}+\mathrm{S}_{3}+\mathrm{G}$ types.

Type II looks like the $S_{1}+G$ type and both Onaka (1949) and Saiki and Ono (1971) confirmed that Cercidiphyllaceae belonged to this type. Type III is similar to the $\mathrm{S}_{1}+\mathrm{S}_{2}+\mathrm{G}$ type which is the most common type. Onaka (1949) cites among other families the Salicaceae (at that time only including the genera Salix and Populus). However, type I cannot correspond to the $\mathrm{S}_{1}+\mathrm{S}_{2}+\mathrm{S}_{3}+\mathrm{G}$ type. Onaka (1949) described "a lessening of the degree of lignification towards the center, and a gradual change to the gelatinous layer."

Several species have been classified in the three classes of eccentricity, or in two types of G-layers. In most cases, eccentricity is an efficient way to detect the formation of tension wood. However, a tree forming tension wood specifically on one side of the stem does not systematically show clear eccentricity, as is the case in Castanea sativa (fig. 9.1 in Gardiner et al. 2014b) or Bagassa guianensis (Bossu 2015). Furthermore, tension wood serves the biological function of controlling tree posture and may consequently be found in several parts of the trunk depending on the past history of the tree and the need to reorient the tree axis. Using eccentricity to predict tension wood is thus less easy, which is why eccentricity should be used with caution when classifying tension wood. 
After Onaka (1949) the diversity of tension wood was classified more simply, as either forming G-layers or lacking them, which facilitated the comparison of different studies. Höster and Liese (1966) surveyed the branches of 110 tropical and temperate tree species, and found $75 \%$ had G-layers in their tension wood, compared to $35 \%$ for shrubs. In tilted branches or young axes, only $46 \%$ of the 122 species studied by Fisher and Stevenson (1981) presented tension wood with G-layers. Among buttresses and aerial roots of 48 tropical species, however, only $10 \%$ had distinct G-layers (Fisher 1982). No G-layer was found in nine Japanese shrub species (Sultana et al. 2010). However, do shrubs, branches, buttresses and aerial roots produce high tensile stresses? Preliminary studies suggest that tensile stress in buttresses correspond to those in normal wood (fig. 5.6 in Fournier et al. 2014). Finally, in a study on 21 tropical species from 18 families, $38 \%$ were shown to have a G-layer in their tension wood despite the higher growth stresses recorded on the tension wood side of all the trees considered (Clair et al. 2006). Thus, although tension wood with a G-layer has been more widely studied, it is much less common than tension wood without a G-layer.

\section{Unusual observations of tension wood reported in the literature}

Figure 2 gives a schematic representation of the anatomy of tension wood fibres described or deduced from the literature. All the observations presented in the text are summarized in Table 1.

Simarouba amara: from lacking a G-layer to having a G-layer with late lignification

The tension wood of several species without a G-layer has been characterised thanks to measurements of growth stress combined with anatomical observations. Amongst

(text continued on page 254)
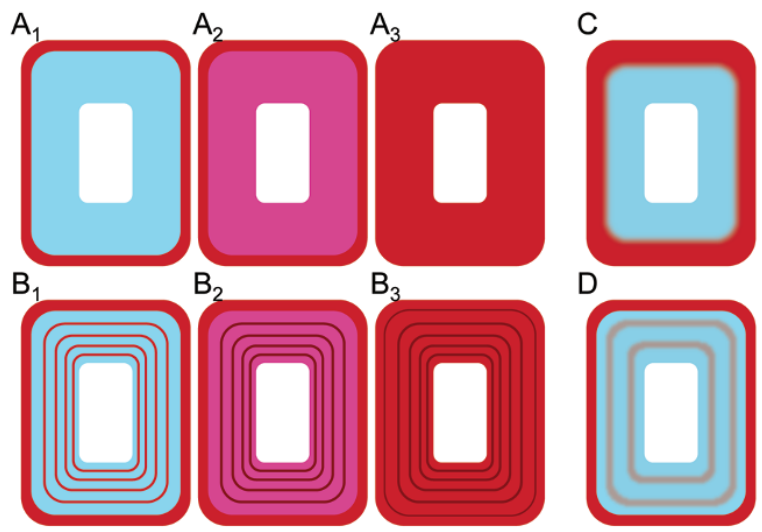

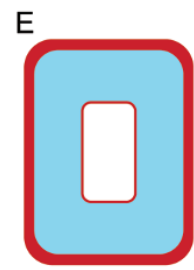

F

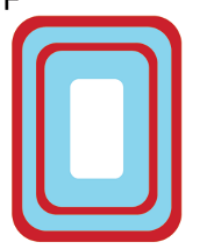

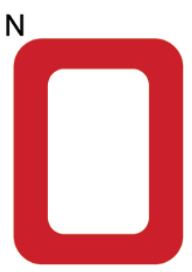

Figure 2. Schematic representation of the anatomy of tension wood fibres presenting $S$ and G-layer ( $\mathrm{S}_{1}$ layer and $\mathrm{S}_{2}$ layer - if any - are presented as a single layer) (A-F), compared to a normal wood fibre $(N) .-A_{1}-A_{3}$ : Unlignified to highly lignified G-layer. $-B_{1}-B_{3}$ : Unlignified to highly lignified multi-layered G-layer. $-\mathrm{C}$ : Progressive decrease in lignin content from the $\mathrm{S}_{1}$ or the $\mathrm{S}_{2}$ layer to the G-layer. - D: Concentric lignin deposition in the G-layer. - E: Inner thin lignified ring in the G-layer. $-\mathrm{F}$ : Thick lignified ring inside the G-layer. $-\mathrm{N}$ : Normal wood fibre, including $\mathrm{S}_{1}, \mathrm{~S}_{2}, \mathrm{~S}_{3}$. 
Table 1. Summary of the tension wood type, sampling location, tilting condition and occurrence of stress measurement in the cited literature.

Axes: $\mathrm{T}=$ trunk, $\mathrm{B}=$ branch, $\mathrm{Bu}=$ buttress, $\mathrm{Bo}=$ board, $\mathrm{HW}=$ heartwood. - Tilting condition: $\mathrm{A}=$ artificially tilted, $\mathrm{N}=$ naturally tilted, $\mathrm{S}=$ straight tree, $\mathrm{NM}=$ tension wood not mentioned. - Stress meas.: $\mathrm{Y}$ indicates a study where measurements of maturation stress were performed. - Classif. Fig. 2: Tension wood type (A-F) is given according to the classification pictured in Figure 2; $\mathrm{H}=$ tension wood without G-layer. Letters in parentheses refer to less common types. Question marks indicate uncertain classification. - References *: additional observations to the cited study.

\begin{tabular}{|c|c|c|c|c|c|c|}
\hline Family & Species & Axes & $\begin{array}{c}\text { Tilting } \\
\text { condition }\end{array}$ & $\begin{array}{l}\text { Stress } \\
\text { meas. }\end{array}$ & $\begin{array}{l}\text { Classif. } \\
\text { Fig. } 2\end{array}$ & References \\
\hline Altingiaceae & $\begin{array}{l}\text { Liquidambar } \\
\text { styraciflua }\end{array}$ & $\mathrm{T}$ & $\mathrm{N}$ & & $\mathrm{A}_{1}$ & Bowling \& Vaughn 2008 \\
\hline Annonaceae & Annona & $\mathrm{B}, \mathrm{T}$ & $\mathrm{N}, \mathrm{S}$ & & $\mathrm{H}$ ? & Fisher \& Stevenson 1981 \\
\hline Annonaceae & Annona & & & & $\mathrm{H}$ & Onaka 1949 \\
\hline Betulaceae & Alnus pendula & $\mathrm{T}$ & A & & $\mathrm{C}$ & Araki et al. 1983 \\
\hline Bignoniaceae & Catalpa & $\mathrm{B}, \mathrm{T}$ & $\mathrm{N}, \mathrm{S}$ & & $\mathrm{H} ?$ & Fisher \& Stevenson 1981 \\
\hline Cannabaceae & Celtis sinensis & $\mathrm{T}$ & $\mathrm{N}$ & & $\mathrm{A}_{1}$ & Bowling \& Vaughn 2008 \\
\hline Cannabaceae & Celtis sinensis & $\mathrm{B}, \mathrm{T}$ & $\mathrm{N}$ & & $\mathrm{A}_{1}$ & Saiki \& Ono 1971 \\
\hline Casuarinaceae & $\begin{array}{l}\text { Casuarina } \\
\text { equisetifolia }\end{array}$ & & & & $\mathrm{F} ?$ & Sanio 1863 \\
\hline Cercidiphyllaceae & $\begin{array}{l}\text { Cercidiphyllum } \\
\text { japonicum }\end{array}$ & $\mathrm{T}$ & A & & $\mathrm{A}(\mathrm{C})$ & Araki et al. 1983 \\
\hline Cercidiphyllaceae & $\begin{array}{l}\text { Cercidiphyllum } \\
\text { japonicum }\end{array}$ & $\mathrm{B}, \mathrm{T}$ & $\mathrm{N}$ & & $\mathrm{A}(\mathrm{C})$ & Saiki \& Ono 1971 \\
\hline Cornaceae & $\begin{array}{l}\text { Cornus } \\
\text { controversa }\end{array}$ & $\mathrm{T}$ & A & & $\mathrm{A}(\mathrm{C})$ & Araki et al. 1983 \\
\hline Daphnaceae & Daphne odora & & & & $\mathrm{H}$ & Onaka 1949 \\
\hline Euphorbiaceae & $\begin{array}{l}\text { Hevea } \\
\text { brasiliensis }\end{array}$ & $\mathrm{T}$ & & & $A \& B$ & Encinas \& Daniel 1997 \\
\hline Euphorbiaceae & $\begin{array}{l}\text { Hevea } \\
\text { brasiliensis }\end{array}$ & $\mathrm{T}$ & $\mathrm{N}$ & & B & Sujan et al. 2015 \\
\hline Euphorbiaceae & $\begin{array}{l}\text { Mallotus } \\
\text { japonicus }\end{array}$ & $\mathrm{T}$ & A & & B & Nakagawa et al. 2012 \\
\hline Fabaceae & $\begin{array}{l}\text { Caragana } \\
\text { korshinskii }\end{array}$ & $\mathrm{T}$ & S & & $\mathrm{A}_{1}, \mathrm{~A}_{2}, \mathrm{~A}_{3}$ & Furukawa \& Ohashi 1999 \\
\hline Fabaceae & $\begin{array}{l}\text { Carmichaelia } \\
\text { arborea }\end{array}$ & & & & B & Butterfield \& Meylan 1980 \\
\hline Fabaceae & $\begin{array}{l}\text { Hedysarum } \\
\text { scoparium }\end{array}$ & $\mathrm{T}$ & S & & $\mathrm{A}_{1}, \mathrm{~A}_{2}, \mathrm{~A}_{3}$ & Furukawa \& Ohashi 1999 \\
\hline Fabaceae & Inga marginata & $\mathrm{T}$ & $\mathrm{N}$ & $\mathrm{Y}$ & $\mathrm{A}_{1} \& \mathrm{~A}_{3}$ & Clair et al. 2006 \\
\hline Fabaceae & Inga marginata & $\mathrm{T}$ & $\mathrm{N}$ & $\mathrm{Y}$ & $\mathrm{A}_{1} \& \mathrm{~A}_{3}$ & Ruelle et al. 2006 \\
\hline Fabaceae & $\begin{array}{l}\text { Koompassia } \\
\text { malaccensis }\end{array}$ & $\mathrm{T}, \mathrm{HW}$ & & & B & Schmitt et al. 1995 \\
\hline Fabaceae & $\begin{array}{l}\text { Koompassia } \\
\text { malaccensis }\end{array}$ & Bo, HW & $\mathrm{NM}$ & & B & Singh et al. 2004 \\
\hline Fabaceae & $\begin{array}{l}\text { Robinia } \\
\text { pseudoacacia }\end{array}$ & $\mathrm{T}$ & A & & $\mathrm{A}_{1}\left(\mathrm{~B}_{1}\right.$ or $\left.\mathrm{D}\right)$ & Araki et al. 1982 \\
\hline Fabaceae & $\begin{array}{l}\text { Robinia } \\
\text { pseudoacacia }\end{array}$ & $\mathrm{T}$ & A & & $\mathrm{E}$ & Araki et al. 1982 \\
\hline Fabaceae & $\begin{array}{l}\text { Robinia } \\
\text { pseudoacacia }\end{array}$ & $\mathrm{B}, \mathrm{T}$ & $\mathrm{N}$ & & $A_{1}(F)$ & Saiki \& Ono 1971 \\
\hline Fagaceae & Fagus crenata & $\mathrm{B}, \mathrm{T}$ & $\mathrm{N}$ & & $\mathrm{E}$ & Saiki \& Ono 1971 \\
\hline
\end{tabular}


Table 1 continued)

\begin{tabular}{|c|c|c|c|c|c|c|}
\hline Family & Species & Axes & $\begin{array}{l}\text { Tilting } \\
\text { condition }\end{array}$ & $\begin{array}{l}\text { Stress } \\
\text { meas. }\end{array}$ & $\begin{array}{l}\text { Classif. } \\
\text { Fig. } 2\end{array}$ & References \\
\hline Fagaceae & Fagus sylvatica & & & & B ? & Wardrop \& Dadswell 1955 \\
\hline Fagaceae & Fagus sylvatica & & & & A (B ?) & Wardrop 1964 \\
\hline Fagaceae & Fagus sylvatica & $\mathrm{T}$ & $\mathrm{N}$ & & $\mathrm{A} 1 \& \mathrm{E}$ & Lehringer et al. 2009 \\
\hline Fagaceae & Fagus sylvatica & $\mathrm{T}$ & $\mathrm{N}$ & & A1 (A2 \& A3) & Schwarze \& Fink 1998 \\
\hline Fagaceae & Quercus ilex & $\mathrm{T}$ & A & & $\mathrm{A} 1 \& \mathrm{~A} 2$ & Gartner et al. 2003 \\
\hline Fagaceae & Quercus lyrata & Bo & & & $A \& E$ & Wahlgren 1957 \\
\hline Fagaceae & Quercus robur & $\mathrm{B}, \mathrm{T}$ & & & $\mathrm{D}$ & Casperson 1967 \\
\hline Fagaceae & Quercus robur & B & & & $\mathrm{E}$ & Lehringer et al. 2008 \\
\hline Fagaceae & Quercus robur & B & & & $\mathrm{D}$ & Lehringer et al. 2009 \\
\hline Irvingiaceae & Irvingia & $\mathrm{Bu}$ & & & A3 ? & Fisher 1982 \\
\hline Lauraceae & $\begin{array}{l}\text { Ocotea } \\
\text { indirectinervia }\end{array}$ & $\mathrm{T}$ & $\mathrm{N}$ & Y & $\mathrm{A} 1 \& \mathrm{~A} 3$ & Ruelle et al. 2006 \\
\hline Lauraceae & Sextonia rubra & $\mathrm{T}$ & $\mathrm{N}$ & $\mathrm{Y}$ & A1 (B1) & Chang et al. 2009* \\
\hline Lauraceae & Sextonia rubra & Bo & & & $\mathrm{A} 1(\mathrm{~F})$ & Jutte 1956 \\
\hline Lecythidaceae & $\begin{array}{l}\text { Eschweilera } \\
\text { sagotiana }\end{array}$ & $\mathrm{T}$ & $\mathrm{N}$ & $\mathrm{Y}$ & A3 & Clair et al. 2006 \\
\hline Lecythidaceae & $\begin{array}{l}\text { Eschweilera } \\
\text { sagotiana }\end{array}$ & $\mathrm{T}$ & $\mathrm{N}$ & $\mathrm{Y}$ & A3 & Ruelle et al. 2006 \\
\hline Lecythidaceae & Eschweilera sp. & $\mathrm{Bu}$ & & & $\mathrm{A} 1 \& \mathrm{~A} 3$ & Fisher 1982 \\
\hline Magnoliaceae & $\begin{array}{l}\text { Liriodendron } \\
\text { tulipifera }\end{array}$ & $\mathrm{T}$ & A & & A3? & Hiraiwa et al. 2014 \\
\hline Magnoliaceae & $\begin{array}{l}\text { Liriodendron } \\
\text { tulipifera }\end{array}$ & $\mathrm{T}$ & & $\mathrm{Y}$ & A3? & Okuyama et al. 1994 \\
\hline Magnoliaceae & $\begin{array}{l}\text { Liriodendron } \\
\text { tulipifera }\end{array}$ & $\mathrm{T}$ & $\mathrm{N}$ & Y & A3? & Yoshida et al. 2002 \\
\hline Magnoliaceae & Magnolia kobus & $\mathrm{B}$ & & & A3? & Yoshizawa et al. 2000 \\
\hline Magnoliaceae & Magnolia obovata & $\mathrm{T}$ & A & & A3? & Yoshizawa et al. 2000 \\
\hline Magnoliaceae & $\begin{array}{l}\text { Magnolia verecunda, } \\
\text { Michelia compressa }\end{array}$ & & & & $\mathrm{H}$ ? & Onaka 1949 \\
\hline Malvaceae & $\begin{array}{l}\text { Apeiba, } \\
\text { Heliocarpus, Tilia }\end{array}$ & $\mathrm{B}, \mathrm{T}$ & $\mathrm{N}, \mathrm{S}$ & & $\mathrm{H}$ & Fisher \& Stevenson 1981 \\
\hline Malvaceae & Entelea arborescens & & & & $\mathrm{H}$ & Patel 1988 \\
\hline Malvaceae & Lagunaria patersonii & $\mathrm{T}$ & $\mathrm{A}$ & & $\mathrm{H}$ & Bland \& Scurfield 1964 \\
\hline Malvaceae & Lagunaria patersonii & & & & $\mathrm{H}$ & Scurfield 1973 \\
\hline Malvaceae & $\begin{array}{l}\text { Ochroma, } \\
\text { Durio zibethinus }\end{array}$ & $\mathrm{B}, \mathrm{T}$ & $\mathrm{N}, \mathrm{S}$ & & $\mathrm{H}$ ? & Fisher \& Stevenson 1981 \\
\hline Malvaceae & Tilia cordata & B & $\mathrm{N}$ & & $\mathrm{H}$ & Böhlmann 1971 \\
\hline Malvaceae & $\begin{array}{l}\text { Tilia, Hibiscus } \\
\text { syriacus, Firmania } \\
\text { platanifolia }\end{array}$ & & & & $\mathrm{H}$ & Onaka 1949 \\
\hline Myrtaceae & Eucalyptus andreana & $\mathrm{T}$ & $\mathrm{S}$ & $\mathrm{Y}$ & $\mathrm{A} 1, \mathrm{~A} 2, \mathrm{~A} 3$ & Hirohashi et al. 2012 \\
\hline Myrtaceae & Eucalyptus bicostata & $\mathrm{T}$ & A & & $\mathrm{A}(\mathrm{B}$ or $\mathrm{D})$ & Scurfield 1972 \\
\hline Myrtaceae & $\begin{array}{l}\text { Eucalyptus } \\
\text { camaldulensis }\end{array}$ & $\mathrm{T}$ & A & & $\mathrm{A}(\mathrm{B}$ or $\mathrm{D})$ & Bland \& Scurfield 1964 \\
\hline Myrtaceae & $\begin{array}{l}\text { Eucalyptus } \\
\text { elaeophora }\end{array}$ & & & & E & Wardrop 1964 \\
\hline Myrtaceae & Eucalyptus maidenii & $\mathrm{T}$ & $S$ & $\mathrm{Y}$ & $\mathrm{A} 1, \mathrm{~A} 2, \mathrm{~A} 3$ & Hirohashi et al. 2012 \\
\hline Myrtaceae & Eucalyptus nitens & $\mathrm{T}$ & $\mathrm{N}, \mathrm{S}$ & $\mathrm{Y}$ & $\mathrm{A} 1, \mathrm{~A} 2, \mathrm{~A} 3$ & Clair et al. 2013* \\
\hline Myrtaceae & Eucalyptus regnans & $\mathrm{T}$ & S & $\mathrm{Y}$ & A3? & Chafe 1977 \\
\hline
\end{tabular}


(Table 1 continued)

\begin{tabular}{|c|c|c|c|c|c|c|}
\hline Family & Species & Axes & $\begin{array}{c}\text { Tilting } \\
\text { condition }\end{array}$ & $\begin{array}{l}\text { Stress } \\
\text { meas. }\end{array}$ & $\begin{array}{l}\text { Classif. } \\
\text { Fig. } 2\end{array}$ & References \\
\hline Myrtaceae & Eucalyptus smithii & $\mathrm{T}$ & $\mathrm{S}$ & $\mathrm{Y}$ & $\mathrm{A} 1, \mathrm{~A} 2, \mathrm{~A} 3$ & Hirohashi et al. 2012 \\
\hline Myrtaceae & Eucalyptus sp. & $\mathrm{T}$ & A & & $\mathrm{A} 1 \& \mathrm{~A} 2 ?$ & Scurfield \& Wardrop 1963 \\
\hline Myrtaceae & $\begin{array}{l}\text { Lophostemon } \\
\text { confertus }\end{array}$ & $\mathrm{T}$ & A & & $\mathrm{B} ?$ & Scurfield \& Wardrop 1963 \\
\hline Myrtaceae & $\begin{array}{l}\text { Lophostemon } \\
\text { confertus }\end{array}$ & $\mathrm{T}$ & & & $\mathrm{B}$ ? & Scurfield 1973 \\
\hline Myrtaceae & $\begin{array}{l}\text { Lophostemon } \\
\text { confertus }\end{array}$ & & & & $\mathrm{B}$ ? & Wardrop 1964 \\
\hline Oleaceae & $\begin{array}{l}\text { Fraxinus } \\
\text { sieboldiana }\end{array}$ & $\mathrm{B}, \mathrm{T}$ & $\mathrm{N}$ & & $\mathrm{A}(\mathrm{C})$ & Saiki \& Ono 1971 \\
\hline Oleaceae & $\begin{array}{l}\text { Osmanthus } \\
\text { fragrans }\end{array}$ & $\mathrm{T}$ & A & & A3? & Hiraiwa et al. 2007 \\
\hline Oxalidaceae & Averrhoa & $\mathrm{B}, \mathrm{T}$ & $\mathrm{N}, \mathrm{S}$ & & $\mathrm{H}$ & Fisher \& Stevenson 1981 \\
\hline Proteaceae & Grevillea robusta & $\mathrm{T}$ & A & & $\mathrm{B}$ ? & Scurfield \& Wardrop 1963 \\
\hline Proteaceae & Grevillea robusta & $\mathrm{T}$ & & & $\mathrm{B}$ ? & Scurfield 1973 \\
\hline Proteaceae & Grevillea robusta & & & & $\mathrm{B}$ ? & Wardrop 1964 \\
\hline Proteaceae & Hakea laurina & $\mathrm{T}$ & A & & $\mathrm{A} 1 \& \mathrm{~A} 2 ?$ & Scurfield \& Wardrop 1963 \\
\hline Salicaceae & Casearia javitensis & $\mathrm{T}$ & $\mathrm{N}$ & $\mathrm{Y}$ & B & Clair et al. 2006 \\
\hline Salicaceae & $\begin{array}{l}\text { Homalium } \\
\text { foetidum }\end{array}$ & & NM & & $\mathrm{B}$ & Bailey \& Kerr 1935 \\
\hline Salicaceae & $\begin{array}{l}\text { Homalium } \\
\text { luzoniense }\end{array}$ & Bo & NM & & B & Daniel \& Nilsson 1996 \\
\hline Salicaceae & Laetia procera & $\mathrm{T}$ & $\mathrm{N}$ & $\mathrm{Y}$ & $\mathrm{B} 1 \& \mathrm{~B} 2$ & Ruelle et al. 2007c \\
\hline Salicaceae & $\begin{array}{l}\text { Olmediella } \\
\text { betschleriana }\end{array}$ & Bo & $\mathrm{NM}$ & & B & Daniel \& Nilsson 1996 \\
\hline Salicaceae & $\begin{array}{l}\text { Populus } \\
\times \text { euramericana }\end{array}$ & $\mathrm{T}$ & $\mathrm{N}, \mathrm{S}$ & & $\mathrm{A} 1$ & Badia et al. 2006 \\
\hline Salicaceae & Populus deltoides & $\mathrm{T}$ & & & A1 ? & Joseleau et al. 2004 \\
\hline Salicaceae & $\begin{array}{l}\text { Populus deltoides } \\
\times \text { nigra }\end{array}$ & $\mathrm{T}$ & $\mathrm{N}$ & & A1 & Chang et al. 2015 \\
\hline Salicaceae & $\begin{array}{l}\text { Populus deltoides } \\
\times \text { nigra }\end{array}$ & $\mathrm{T}$ & A & & $\mathrm{A} 1$ & Yoshinaga et al. 2012 \\
\hline Salicaceae & $\begin{array}{l}\text { Populus } \\
\text { euramericana }\end{array}$ & $\mathrm{T}$ & A & & A1 & Fujita et al. 1974 \\
\hline Salicaceae & Populus I4551 & $\mathrm{T}$ & $\mathrm{N}$ & $\mathrm{Y}$ & A1 & Fang et al. 2007 \\
\hline Salicaceae & $\begin{array}{l}\text { Populus nigra } \\
\times \text { deltoides }\end{array}$ & $\mathrm{T}$ & A & & $\mathrm{E}$ & $\begin{array}{l}\text { Gierlinger \& Schwanniger } \\
2006\end{array}$ \\
\hline Salicaceae & Populus sp. & & & & A1 & Pilate et al. 2004 \\
\hline Salicaceae & Populus tremula & $\mathrm{T}$ & $\mathrm{N}$ & & A1 & Kim \& Daniel 2012 \\
\hline Salicaceae & Populus tremula & $\mathrm{T}$ & $\mathrm{N}$ & & $\mathrm{A} 1$ & Norberg \& Meier 1966 \\
\hline Salicaceae & $\begin{array}{l}\text { Populus tremula, } \\
\text { P. alba }\end{array}$ & $\mathrm{B}, \mathrm{T}$ & $\mathrm{N}$ & & A1 & Nishikubo et al. 2007 \\
\hline Salicaceae & $\begin{array}{l}\text { Populus tremula } \\
\times \text { alba }\end{array}$ & $\mathrm{T}$ & A & & A & Lafarguette et al. 2004 \\
\hline Salicaceae & $\begin{array}{l}\text { Populus tremula } \\
\times \text { tremuloides }\end{array}$ & $\mathrm{T}$ & $\mathrm{N}$ & & A1 & Chaffey 2000 \\
\hline Salicaceae & $\begin{array}{l}\text { Populus tremula } \\
\times \text { tremuloides }\end{array}$ & $\mathrm{T}$ & $\mathrm{N}$ & & A1 & Daniel et al. 2006 \\
\hline Salicaceae & $\begin{array}{l}\text { Populus tremula } \\
\times \text { tremuloides }\end{array}$ & $\mathrm{T}$ & A & & A1 & Nishikubo et al. 2007 \\
\hline
\end{tabular}




\begin{tabular}{|c|c|c|c|c|c|c|}
\hline Family & Species & Axes & $\begin{array}{l}\text { Tilting } \\
\text { condition }\end{array}$ & $\begin{array}{l}\text { Stress } \\
\text { meas. }\end{array}$ & $\begin{array}{c}\text { Classif. } \\
\text { Fig. } 2\end{array}$ & References \\
\hline Salicaceae & $\begin{array}{l}\text { Populus tremula } \\
\times \text { tremuloides, } \\
\text { Populus tremula }\end{array}$ & $\mathrm{T}$ & A & & A1 & $\begin{array}{l}\text { Andersson-Gunnerås et al } \\
2006\end{array}$ \\
\hline Salicaceae & Salix lucida & $\mathrm{B}, \mathrm{T}$ & $\mathrm{N}$ & & $\mathrm{A} 1, \mathrm{~A} 2 \& \mathrm{~A} 3$ & Ghislain et al. 2016 \\
\hline Sapindaceae & Acer & Bo & & & $\mathrm{A} 1, \mathrm{~B} 1 \& \mathrm{E}$ & Lehringer et al. 2009 \\
\hline Sapindaceae & Acer & $\mathrm{T}$ & $S$ & & A (B?) & Morey \& Cronshaw 1968 \\
\hline Sapindaceae & $\begin{array}{l}\text { Aesculus } \\
\text { hippocastanum }\end{array}$ & & & & $\mathrm{D}$ & Casperson 1961 \\
\hline Simaroubaceae & Simarouba amara & $\mathrm{T}$ & $\mathrm{A}, \mathrm{N}$ & & A3 & Roussel \& Clair 2015 \\
\hline Siparunaceae & Siparuna bifida & & NM & & $\mathrm{D}$ & Bailey \& Kerr 1935 \\
\hline Tetrameristaceae & Tetramerista glabra & & NM & & $\mathrm{D}$ & Bailey \& Kerr 1935 \\
\hline Thymelaceae & $\begin{array}{l}\text { Wikstroemia tricho- } \\
\text { toma, Edgeworthia } \\
\text { papyrifera }\end{array}$ & & & & $\mathrm{H}$ & Onaka 1949 \\
\hline Trochodendraceae & $\begin{array}{l}\text { Trochodendron } \\
\text { aralioides }\end{array}$ & $\mathrm{T}$ & A & & $\mathrm{A} 1 \& \mathrm{~B} 1$ & Hiraiwa et al. 2013 \\
\hline
\end{tabular}

these species, Simarouba amara has been extensively studied (Ruelle 2006; Ruelle et al.2007a, 2007b, 2011). Strong tensile stress was recorded on the upper side of leaning trunks, but neither the development of G-layers, nor a difference in cell wall thickness was observed. The angle of the microfibrils of cellulose and the size of cellulose aggregates were the only parameters which differed between opposite wood and tension wood (Ruelle et al. 2007b).

However, Roussel and Clair (2015) recently demonstrated that in Simarouba amara tension wood, a G-layer is visible during cell wall maturation, and that it becomes lignified at a later stage than in normal wood. The G-layer starts its lignification after more than 60 days of tilting in saplings. These authors also documented other species with similar patterns. This means that the usual unlignified nature of the G-layer may be masked by late deposition of lignin, and that the usual staining no longer identifies tension wood fibres. Therefore, to assess the occurrence of a G-layer in tension wood, one needs to look at the maturation process. The tension wood of Simarouba amara, thought to be without a G-layer, is now known to develop a G-layer, which later becomes lignified (Fig. 2A).

\section{Laetia procera: multi-layered G-layers}

A peculiar multi-layered fibre structure has been described in the xylem fibres of Salicaceae sensu lato: Homalium foetidum, H. luzoniense and Olmediella betschleriana (Bailey \& Kerr 1935; Daniel \& Nilsson 1996). A similar multi-layered structure with an " $\mathrm{S}_{2}$ " composed of two to five thick and thin layers were observed in the fibre walls in a heartwood sample of a member of the Fabaceae, Koompassia malaccensis (Schmitt et al. 1995; Singh et al. 2004). This multi-layered structure was later linked to the occurrence of tension wood in Casearia javitensis and Laetia procera thanks to measurements of maturation strain (Clair et al. 2006; Ruelle et al. 2007c). These multi-layered fibres occur in the tension wood of most of the former Flacourtiaceae 
now classified as Salicaceae sensu lato (Ghislain et al.2016). They not only occur in tension wood but have also been reported in reaction phloem fibres (Nanko et al. 1982; Nakagawa et al.2012,2014). The fibre cell wall is composed of thick layers with a low cellulose microfibril angle separated by thin lignified layers. This peculiar tension wood has been reported in Carmichaelia arborea (Fabaceae) (Butterfield \& Meylan 1980), Hevea brasiliensis (Euphorbiaceae) (Encinas \& Daniel 1997) and Mallotus japonicus (Euphorbiaceae) (Nakagawa et al. 2012).

\section{Early reports of lignin deposition in the G-layer: what do we know?}

Even though the presence of lignin in the G-layer has long been rejected in the literature, its occurrence has nevertheless been suggested several times. Bailey and Kerr (1935) already described lignin deposition in the G-layer, even if this layer was not yet related to tension wood. Jutte (1956) discussed the definition of G-layers as being unlignified. Casperson (1967) described lignin deposition in G-layers of tension wood.

We reviewed all the reports of lignin in G-layers. It is always important to consider the sampling location in the sapwood or heartwood, which is, alas, not always specified in the papers we reviewed. It is important since the cellulose/lignin staining used to locate G-layers is not specific to lignin but may also stain other polyphenolic compounds, especially from heartwood extractives which have to be distinguished from lignin deposited in the G-layer during maturation, in the vicinity of the cambium. It is known that the G-layer of Fagus sylvatica can be encrusted by lignin or other polyphenolic compounds during the formation of the false heartwood (Baum et al. 2000) and the G-layer of Schinopsis balansae (Anacardiaceae) has been reported to display encrustations of tannins during heartwood formation (Streit \& Fengel 1995). So particular care should be taken when tension wood is taken from a piece of wood in the absence of any other specification concerning its location.

\section{More lignin in the latewood of temperate species}

In temperate species, only traces of lignin have been found in the G-layer of Populus deltoides tension wood (Joseleau et al.2004). However, a larger proportion of lignin is present in the G-layer of latewood fibres: its deposition in the G-layer of Quercus robur (Fagaceae) is either scattered or concentric (Casperson 1967) while it is confined to the outer part of the G-layer in the latewood of Cercidiphyllum japonicum and Fraxinus sieboldiana (Saiki \& Ono 1971).This is why the two last rows of latewood cells in a tension wood sample of Fagus sylvatica (Fagaceae) presenting a higher content in lignin were said to be normal wood (Schwarze \& Fink 1998). This may also explain why Ruelle (2014) noted that "tension wood is preferentially observed in earlywood of temperate species."

\section{Inner thin lignified layer and outer gradual change from $S_{1}$ to $G$}

A thin inner lignified layer in the G-layer (Fig. 2E) has been observed in the following temperate species: Fagus crenata (Saiki \& Ono 1971), Robinia pseudoacacia (Araki et al. 1982), Quercus lyrata (Wahlgren 1957), Eucalyptus elaeophora (Wardrop 
1964), Populus nigra $\times$ Populus deltoides (Gierlinger \& Schwanninger 2006), Quercus robur (Lehringer et al.2008), Acer sp. and Fagus sylvatica (Lehringer et al. 2009).

Within the G-fibre itself, Prodhan et al. (1995a) proposed two types of microfibril deposition in Fraxinus mandshurica var. japonica. Cellulose microfibrils are progressively deposited from the $S_{2}$ layer to the G-layer with an angle either ceasing at $0^{\circ}$ or passing by $0^{\circ}$ and ending at maximum $25^{\circ}$. Interestingly, in the second type of microfibril deposition, the inner part of the G-layer has a varying angle. In addition, the transition between the $S_{1}$ layer and the G-layer is progressive and the G-layer stained weakly for lignin in this outer zone (Prodhan et al. 1995b; Fig. 2C); this resembles in fact the type I of Onaka (1949), with a gradual change from $\mathrm{S}_{1}$ to G. Saiki and Ono (1971) made a similar observation concerning the G-layer of Fraxinus sieboldiana. Araki et al. (1983) also reported lignin staining in the outer G-layer, in what the authors called the G', which shows a gradual change of the orientation of the microfibrils from an angle similar to the one in an $\mathrm{S}_{2}$ to an angle close to $0^{\circ}$ as in $\mathrm{G}$ in Cercidiphyllum japonicum, Cornus controversa and Alnus pendula.

\section{Broad distribution of lignin in G-layers}

In Lophostemon confertus (Myrtaceae, formerly Tristania conferta), lignin precursors penetrate the G-layer when the fibre is far enough away from the cambium (Scurfield 1973). This author suggested that these lignin precursors may be released during the terminal stage of cell senescence (Scurfield 1972).

Irregular lignin deposition has been observed in the tension wood of Eucalyptus sp. (Myrtaceae) and Hakea laurina (Proteaceae) (Scurfield \& Wardrop 1963). Some tension wood cells differ in the intensity of deposition. Only some G-layers of Salix lucida (Salicaceae) stained strongly for lignin while, in some other species, all the G-layers stained for lignin (Ghislain et al. 2016).

G-layers are also present in various shrubby Fabaceae. In Caragana korshinskii and Hedysarum scoparium three types of G-layers in tension wood have been distinguished: unlignified, partly lignified and lignified (Furukawa \& Ohashi 1999). Lignified G-layers have also been described in Ephedra aspera and E. viridis (Ephedraceae), although the authors doubt their role in stem reorientation (Montes et al. 2015). The lignin content in the G-layer of these shrubs increases with stem age.

\section{Multi-layered fibre walls and lignification}

Jutte (1956) observed tension wood with abnormal fibres in a board of Ocotea rubra (currently Sextonia rubra, Lauraceae). This author defined three types of tension wood fibres. Type I: fibres with an inner unlignified layer (G-layer). Type II: fibres like type I but with an additional inner lignified layer which can be thick or thin. Type III: fibres like fibre II but with an additional inner unlignified layer. In this species, Chang et al. (2009) described the tension wood as having a thick unlignified G-layer. In the same sample, we also observed a few fibres exhibiting unlignified multi-layered G-layers (additional observation to the study published by Chang et al. 2009).

Ruelle et al. (2007c) showed that some multi-layered G-layers of Laetia procera are slightly lignified in some fibres and not in others in the same portion of tension wood. 
Ghislain et al. (2016) added that in some species all multi-layered G-layers become lignified (Fig. 2B). But types II and III of Jutte (1956) are the first reports of lignification in only one thick layer while others remained unlignified. As Jutte (1956) did not mention where in the tree the samples came from, we do not know if this observation can be attributed to heartwood formation.

In tension wood of Robinia pseudoacacia thin concentric rings within G-layers were stained with $\mathrm{KMnO}_{4}$ and divided into two or more sublayers (Araki et al. 1982). In this species, an extraordinary sublayer, thicker than the thin layer in a multi-layered G-layer, was stained with $\mathrm{KMnO}_{4}$ (Saiki \& Ono 1971; Fig. 2F). The authors do not specify if the fibre was located in latewood and/or heartwood. A lignified ring has been observed in the G-layer of Casuarina equisetifolia (Casuarinaceae) (Sanio 1863). The G-layer of Eucalyptus camaldulensis (Myrtaceae) is divided into two sublayers by a thin lignified ring (Bland \& Scurfield 1964).

\section{Does tension wood lacking G-layers really exist?}

Do some species develop a kind of tension wood, with no unlignified or lignified G-layers? Onaka (1949) described tension wood with thin cell walls and no difference in lignification from normal wood in Annona (Annonaceae), Firmania platanifolia, Hibiscus syriacus, Tilia (Malvaceae), Daphne odora (Daphnaceae), Edgeworthia papyrifera and Wikstroemia trichotoma (Thymelaeaceae), Liriodendron tulipifera, Magnolia verecunda, and Michelia compressa (Magnoliaceae). However, in recent observations on Liriodendron tulipifera (Hiraiwa et al. 2014) the cell wall in tension wood was reported to be rather thick, so this sample probably had G-layers masked by late lignification.

Lagunaria patersonii (Malvaceae) has thinner fibre walls in tension wood than in normal wood fibres (Bland \& Scurfield 1964; Scurfield 1973). Böhlmann (1971) observed Tilia cordata (Malvaceae) and found no G-layers in its tension wood. Instead, this author observed the phloem and proposed a stress generation mechanism based on phloem instead of wood. Indeed, Onaka (1949) observed greater development of bark on the upper side of Tilia and even proposed a class called "eccentricity in wood and bark" (Fig. 1).

Fisher and Stevenson (1981) assumed that the same mechanism of reaction phloem could occur in Apeiba, Heliocarpus, Tilia (Malvaceae), Averrhoa (Oxalidaceae) and possibly in Annona (Annonaceae), Catalpa (Bignoniaceae), Durio zibethinus and Ochroma (Malvaceae).

Furthermore, these authors noted that Cananga (Annonaceae), Alstonia angustiloba (Apocynaceae), Cordia alliodora, C. lutea, C. panamensis (Boraginaceae), Garcinia nigrolineata (Clusiaceae), Apeiba, Durio zibethinus, Ochroma, Sterculia ceramica, S. foetida (Malvaceae), and Hamelia (Rubiaceae) have enlarged unlignified parenchyma in their tension wood. However, Sterculia foetida was said to have either no G-layer or G-layers on the upper side of the leaning branch.

Patel (1988) described a non-homogeneous distribution of what the author called pith-like parenchyma in a tree of Entelea arborescens (Malvaceae) with high eccentricity, and concluded it was reaction wood. 
It therefore appears that tension wood really lacking G-layers does exist, with fibres with thinner cell walls, different distribution/size of parenchyma cells and a thicker phloem. However, growth stress measurements coupled with anatomical studies are necessary to conclude on the mechanism underlying such tension wood lacking a G-layer.

\section{New light on old data}

Acknowledging the occurrence of late lignification in the G-layers of Simarouba amara, of lignin deposits in the G-layer of many species, of multi-layered G-layers, and of tension wood with thin cell walls and no G-layers, throws new light on reports in the literature on tension wood previously considered to be unusual. Indeed, species previously believed to be lacking G-layers might actually have lignified ones, while others really do lack them.

\section{Observations of late lignification such as in tension wood of Simarouba amara}

Unusual fibres of Eschweilera sp. (Lecythidaceae) with delayed lignification of their inner wall were observed by Fisher (1982) in tension wood. Eschweilera sagotiana is not reported to have G-layers although a strong tensile stress has been observed on the upper side of the leaning trunk (Clair et al. 2006; Ruelle et al. 2006). However, the swollen aspect of a G-layer and its weak adherence to outer layers is clearly visible, even though it is lignified. Similarly Irvingia (Irvingiaceae) has been described as having an inner layer that becomes lignified in mature wood but remains separate from the other layers (Fisher 1982). These observations suggest that late lignification masks the expected unlignified aspect of the G-layer as described by Roussel and Clair (2015). Osmanthus fragrans (Oleaceae) tension wood has no G-layer but the cell wall fibres lack an $S_{3}$ and have an $S_{2}$ with a reduced microfibril angle (MFA) and less lignin than normal wood (Hiraiwa et al. 2007). Similar observations have been reported in Magnolia kobus and M. obovata (Magnoliaceae) (Yoshizawa et al. 2000) and Liriodendron tulipifera (Magnoliaceae) (Yoshida et al. 2002; Hiraiwa et al. 2014). These species might thus all have lignified G-layers. Increasing maturation stresses have indeed been linked to decreasing MFA, increasing $\alpha$-cellulose content and increasing cellulose crystallinity in Liriodendron tulipifera (Okuyama et al. 1994).

\section{Irregular deposition of lignin}

Trees of Eucalyptus regnans with high maturation stresses might have gelatinous layers partly hidden by lignification (Chafe 1977). This would explain the anatomical differences with the fibres of normal wood. Although the authors did not describe it in that way, autofluorescent photographs of Eucalyptus andreana, E. maidenii and E. smithii showed the occurrence of unlignified G-layers next to lignified G-layers and partly lignified G-layers, with either the outer or the inner part of the G-layer containing lignin (Hirohashi et al. 2012). Similar observations were made in E. nitens (additional observation to the study published by Clair et al.2013).

A confocal fluorescence micrograph of Populus nigra was overexposed in order to visualize the G-layer, yet it is interesting to note the heterogeneity of staining of the 
G-layer, with the inner part looking brighter (Donaldson 2001). In Ocotea indirectinervia (Lauraceae), lignified G-layers can be seen in the vicinity of unlignified ones (Ruelle et al. 2006). In Inga marginata (Fabaceae), no G-layer was mentioned (Clair et al. 2006; Ruelle et al. 2006), although lignified and unlignified G-layers appear in the figure.

\section{Concentric deposition of lignin and/or multi-layered tension wood fibre walls}

The concentric deposition of aromatic compounds or lignin described in the G-layer of Quercus sp. and Aesculus sp. (Casperson 1961, 1967; Lehringer et al. 2009) appear to be an intermediate stage between the usual G-layer and a multi-layered G-layer in which the alternating deposition of thick and thin layers is regular (Fig. 2D). It is not clear whether Eucalyptus bicostata has multi-layered G-layers in its tension wood or whether it has concentric deposition like in Quercus sp. (Scurfield 1972). In tension wood of Trochodendron aralioides (Trochodendraceae) (fig. 5 in Hiraiwa et al. 2013) a few multi-layered G-layers can be seen among the usual G-layers.

The occurrence of two sublayers in some G-layers of Acer species (Lehringer et al. 2009) can also be observed in some fibres in the figures of Morey and Cronshaw (1968). Two sublayers are also present in the G-layer of Hevea brasiliensis var. Gondang Tapen in which maturation stresses and MFA were measured (Sujan et al. 2015). The lamellae of the G-layer of Lophostemon confertus (Myrtaceae, formerly in Tristania), and Grevillea robusta (Proteaceae) (Scurfield \& Wardrop 1963; Wardrop 1964; Scurfield 1973) appears to correspond to multi-layered tension wood fibres, as does Fagus sylvatica (Wardrop \& Dadswell 1955; Wardrop 1964).

\section{CONCLUSIONS}

After reviewing the diversity of tension wood reported in the literature, we are now able to look at tension wood with fresh eyes, and advances in our understanding of tension wood allow us to re-interpret past observations.

This study confirms that tension wood shows a wide range of anatomical structures which make it impossible to use anatomical criteria to define it, but require instead the use of stress measurement in the living tree. Indeed, tension wood can have lignified or unlignified G-layers or even no G-layer at all. To determine if tension wood has formed, it is thus indispensable to know the history of the tree sampled. In the ideal case, we should work with artificially inclined material. If this is not possible, we should either be able to retrace the history of the material under study or measure its stresses, or at least be aware of the limitations of our methods.

In future studies on tension wood diversity, we will still maintain at least two classes, considering the mechanisms that produce stress. The first group will include species which produce tension wood with G-layers. These G-layers may be unlignified or contain varying amounts of lignin and/or have many layers, even in temperate species. In unlignified G-layers, the generation of stress during maturation was shown to occur during the thickening of the G-layer. When the G-layer is lignified, lignification occurs late, after wall thickening is complete, and one would expect that the maturation stress 
has already been generated and that lignification will not produce additional stresses but only fix the tensile stress while causing reinforcement of the cell wall. In this group, knowledge of the mechanism behind the generation of maturation stress is progressing and poplar will probably continue to be the model used to advance our understanding of its functioning. The second group includes the species with tension wood with fibres which do not produce G-layers, with thin cell walls, and with apparently different size and distribution of parenchyma cells. In these species, the mechanism (or mechanisms) behind the generation of tensile stress remain(s) unknown and studies combining stress measurements and microscopic and ultrastructural development are needed to propose a classification able to incorporate more species.

\section{ACKNOWLEDGEMENTS}

We wish to thank Miho Kojima (Kyoto University) for her help in providing and translating some Japanese papers, and Pieter Baas for the critical reading of an early draft of our manuscript. This work was supported by the French National Research Agency in the framework of the project "StressInTrees" (ANR-12-BS09-0004). BG benefits from an "Investissements d'Avenir" grant managed by the French National Research Agency (CEBA, ANR-10-LABX-25-01).

\section{REFERENCES}

Abedini R, Clair B, Pourtahmasi K, Laurans F, Arnould O. 2015. Cell wall thickening in developing tension wood of artificially bent poplar trees. IAWA J. 36: 44-57.

Andersson-Gunnerås S, Mellerowicz EJ, Love J, Segerman B, Ohmiya Y, Coutinho PM, Nilsson P, Henrissat B, Moritz T, Sundberg B. 2006. Biosynthesis of cellulose-enriched tension wood in Populus: global analysis of transcripts and metabolites identifies biochemical and developmental regulators in secondary wall biosynthesis. Plant J. 45: 144-165.

Araki N, Fujita M, Saiki H, Harada H. 1982. Transition of the fiber wall structure from normal wood to tension wood in Robinia pseudoacacia L. and Populus euramericana Guinier. Mokuzai Gakkaishi 28: 267-273.

Araki N, Fujita M, Saiki H \& Harada H. 1983. Transition of the fiber wall structure from normal wood to tension wood in certain species having $\mathrm{S}_{1}+\mathrm{G}$ and $\mathrm{S}_{1}+\mathrm{S}_{2}+\mathrm{S}_{3}+\mathrm{G}$ types. Mokuzai Gakkaishi 29: 491-499.

Archer RR. 1987. Growth stresses and strains in trees. Springer, Berlin, Heidelberg.

Badia MA, Constant T, Mothe F, Nepveu G. 2006. Tension wood occurrence in three cultivars of Populus $\times$ euramericana. Part I: Inter-clonal and intra-tree variability of tension wood. Ann. For. Sci. 63: 23-30.

Bailey IW, Kerr T. 1935. The visible structure of the secondary wall and its significance in physical and chemical investigations of tracheary cells and fibers. J. Arnold Arbor. 16: 273-300.

Baum S, Schwarze FWMR, Fink S. 2000. Persistence of the gelatinous layer within altered tension-wood fibres of beech degraded by Ustulina deusta. New Phytol. 147: 347-355.

Bland DE, Scurfield G. 1964. The chemistry of reaction wood. IV. The distribution and nature of the lignin in seedlings of hardwoods. Holzforschung 18: 161-166.

Böhlmann D. 1971. Zugbast bei Tilia cordata Mill. Holzforschung 25: 1-4.

Bossu J. 2015. Potentiel de Bagassa guianensis et Cordia alliodora pour la plantation en zone tropicale. Description d'une stratégie de croissance optimale alliant vitesse de croissance et qualité du bois. Thesis, Université de Guyane, Kourou. 331 pp.

Bowling AJ, Vaughn KC. 2008. Immunocytochemical characterization of tension wood: gelatinous fibers contain more than just cellulose. Amer. J. Bot. 95: 655-663. 
Butterfield BG, Meylan BA. 1980. Three-dimensional structure of wood. Springer Science \& Business Media.

Casperson G. 1961. Licht- und elektronenmikroskopische Untersuchungen über den zeitlichen Ablauf der Zellwandbildung bei Laubhölzern. Berichte Dtsch. Bot. Ges. 74: 271-273.

Casperson G. 1967. Über die Bildung von Zellwänden bei Laubhölzern. 4. Mitt.: Untersuchungen an Eiche (Quercus robur L.). Holzforschung 21: 1-6.

Chafe SC. 1977. Radial dislocations in the fibre wall of Eucalyptus regnans trees of high growth stress. Wood Sci. Technol. 11: 59-77.

Chaffey N. 2000. Microfibril orientation in wood cells: new angles on an old topic. Trends Plant Sci. 5: 360-362.

Chang S-S, Clair B, Ruelle J, Beauchêne J, Di Renzo F, Quignard F, Zhao G-J, Yamamoto H, Gril J. 2009. Mesoporosity as a new parameter for understanding tension stress generation in trees. J. Exp. Bot. 60: 3023-3030.

Chang S-S, Quignard F, Alméras T, Clair B. 2015. Mesoporosity changes from cambium to mature tension wood: a new step toward the understanding of maturation stress generation in trees. New Phytol. 205: 1277-1287.

Clair B, Alméras T, Pilate G, Jullien D, Sugiyama J, Riekel C. 2011. Maturation stress generation in poplar tension wood studied by synchrotron radiation microdiffraction. Plant Physiol. 155: 562-570.

Clair B, Alteyrac J, Gronvold A, Espejo J, Chanson B, Alméras T. 2013. Patterns of longitudinal and tangential maturation stresses in Eucalyptus nitens plantation trees. Ann. For. Sci. 70: 801-811.

Clair B, Gril J, Baba K, Thibaut B, Sugiyama J. 2005a. Precautions for the structural analysis of the gelatinous layer in tension wood. IAWA J. 26: 189-195.

Clair B, Gril J, Di Renzo F, Yamamoto H, Quignard F. 2008. Characterization of a gel in the cell wall to elucidate the paradoxical shrinkage of tension wood. Biomacromolecules 9: 494-498.

Clair B, Ruelle J, Beauchêne J, Prévost M-F, Fournier M. 2006. Tension wood and opposite wood in 21 tropical rain forest species. 1. Occurence and efficiency of G-layer. IAWA J. 27: $329-338$.

Clair B, Thibaut B, Sugiyama J. 2005b. On the detachment of the gelatinous layer in tension wood fiber. J. Wood Sci. 51: 218-221.

Daniel G, Filonova L, Kallas ÅM, Teeri TT. 2006. Morphological and chemical characterisation of the G-layer in tension wood fibres of Populus tremula and Betula verrucosa: Labelling with cellulose-binding module CBM1 Hj Cel7A and fluorescence and FE-SEM microscopy. Holzforschung 60: 618-624.

Daniel G, Nilsson T. 1996. Polylaminate concentric cell wall layering in fibres of Homalium foetidum and its effect on degradation by microfungi. In: Third Pacific on Regional Conference on Recent Advances in Wood Anatomy: 369-372. New Zealand Forest Research Institute, Rotorua.

Donaldson LA. 2001. Lignification and lignin topochemistry - an ultrastructural view. Phytochemistry 57: 859-873.

Encinas O, Daniel G. 1997. Degradation of the gelatinous layer in aspen and rubberwood by the blue stain fungus Lasiodiplodia theobromae. IAWA J. 18: 107-115.

Fang C-H, Clair B, Gril J, Alméras T. 2007. Transverse shrinkage in G-fibers as a function of cell wall layering and growth strain. Wood Sci. Technol. 41: 659-671.

Fisher JB. 1982. A survey of buttresses and aerial roots of tropical trees for presence of reaction wood. Biotropica 14: 56.

Fisher JB, Stevenson JW. 1981. Occurrence of reaction wood in branches of dicotyledons and its role in tree architecture. Bot. Gaz. 142: 82-95. 
Fournier M, Alméras T, Clair B, Gril J. 2014. Biomechanical action and biological functions. In: Gardiner B, Barnett J, Saranpää P, Gril J (eds.), The biology of reaction wood: 139-169. Springer, Berlin, Heidelberg.

Fujita M, Harada H, Saiki H. 1974. Electron microscopy of microtubules and cellulose microfibrils in secondary wall formation of poplar tension wood fibers. Mokuzai Gakkaishi 20: $147-156$.

Furukawa I, Ohashi G. 1999. Anatomical features and characteristics of wood quality of leguminous woody xerophytes grown in China deserts. Hardwood Res. 8: 45-51.

Gardiner B, Barnett J, Saranpää P, Gril J. 2014a. The biology of reaction wood. Springer, Berlin, Heidelberg.

Gardiner B, Flatman T, Thibaut B. 2014b. Commercial implications of reaction wood and the influence of forest management. In: Gardiner B, Barnett J, Saranpää P, Gril J (eds.), The biology of reaction wood: 249-274. Springer, Berlin, Heidelberg.

Gartner BL, Roy J, Huc R. 2003. Effects of tension wood on specific conductivity and vulnerability to embolism of Quercus ilex seedlings grown at two atmospheric $\mathrm{CO}_{2}$ concentrations. Tree Physiol. 23: 387-395.

Ghislain B, Nicolini E-A, Romain R, Ruelle J, Yoshinaga A, Alford MH, Clair B. 2016. Multilayered structure of tension wood cell walls in Salicaceae sensu lato and its taxonomic significance. Bot. J. Linn. Soc. 182: 744-756.

Gierlinger N, Schwanninger M. 2006. Chemical imaging of poplar wood cell walls by confocal Raman microscopy. Plant Physiol. 140: 1246-1254.

Hiraiwa T, Aiso H, Ishiguri F, Takashima Y, Iizuka K, Yokota S. 2014. Anatomy and chemical composition of Liriodendron tulipifera stems inclined at different angles. IAWA J. 35: $463-475$.

Hiraiwa T, Toyoizumi T, Yokota S, Ishiguri F, Iizuka K, Yokota S, Yoshizawa N. 2013. Characteristics of Trochodendron aralioides tension wood formed at different inclination angles. IAWA J. 34: 273-284.

Hiraiwa T, Yamamoto Y, Ishiguri F, Izuka K, Yokota S, Yoshizawa N. 2007. Cell wall structure and lignin distribution in the reaction wood fiber of Osmanthus fragrans var. aurantiacus Makino. Cellul. Chem. Technol. 41: 537-543.

Hirohashi A, Kojima M, Yoshida M, Yamamoto H, Watanabe Y, Inoue H, Kamoda S. 2012. Wood properties of 6 fast-growing Eucalyptus species grown in Japan. Mokuzai Gakkaishi 58: 339-346.

Höster H-R, Liese W. 1966. Über das Vorkommen von Reaktionsgewebe in Wurzeln und Ästen der Dikotyledonen. Holzforschung 20: 80-90.

IAWA Committee. 1964. Multilingual glossary of terms used in wood. Verlagsbuchanstalt Konkordia, Winterthur.

Joseleau J-P, Imai T, Kuroda K, Ruel K. 2004. Detection in situ and characterization of lignin in the G-layer of tension wood fibres of Populus deltoides. Planta 219: 338-345.

Jutte SM. 1956. Tension wood in wane (Ocotea rubra Mez). Holzforschung 10: 33-35.

Kim JS, Daniel G. 2012. Distribution of glucomannans and xylans in poplar xylem and their changes under tension stress. Planta 236: 35-50.

Lafarguette F, Leplé J-C, Déjardin A, Laurans F, Costa G, Lesage-Descauses M-C, Pilate G. 2004. Poplar genes encoding fasciclin-like arabinogalactan proteins are highly expressed in tension wood. New Phytol. 164: 107-121.

Lehringer C, Daniel G, Schmitt U. 2009. TEM/FE-SEM studies on tension wood fibres of Acer spp., Fagus sylvatica L. and Quercus robur L. Wood Sci. Technol. 43: 691-702.

Lehringer C, Gierlinger N, Koch G. 2008. Topochemical investigation on tension wood fibres of Acer spp., Fagus sylvatica L. and Quercus robur L. Holzforschung 62: 255-263. 
Metzger K. 1908. Über das Konstruktionsprinzip des sekundären Holzkörpers. Naturwiss. Z. für Forst- und Landwirtsch. 6: 249-278.

Montes MM, Ewers FW, Bobich EG. 2015. Gelatinous fibres are not produced in response to induced stresses in Ephedra. IAWA J. 36: 121-137.

Morey PR, Cronshaw J. 1968. Induction of tension wood by 2,4-dinitrophenol and auxins. Protoplasma 65: 393-405.

Moulia B, Fournier M. 2009. The power and control of gravitropic movements in plants: a biomechanical and systems biology view. J. Exp. Bot. 60: 461-486.

Nakagawa K, Yoshinaga A, Takabe K. 2012. Anatomy and lignin distribution in reaction phloem fibres of several Japanese hardwoods. Ann. Bot. 110: 897-904.

Nakagawa K, Yoshinaga A, Takabe K. 2014. Xylan deposition and lignification in the multilayered cell walls of phloem fibres in Mallotus japonicus (Euphorbiaceae). Tree Physiol. 34: 1018-1029.

Nanko H, Saiki H, Harada H. 1982. Structural modification of secondary phloem fibers in the reaction phloem of Populus euramericana. Mokuzai Gakkaishi 28: 202-207.

Nishikubo N, Awano T, Banasiak A, Bourquin V, Ibatullin F, Funada R, Brumer H, Teeri TT, Hayashi T, Sundberg B, Mellerowicz EJ. 2007. Xyloglucan endo-transglycosylase (XET) functions in gelatinous layers of tension wood fibers in poplar - A glimpse into the mechanism of the balancing act of trees. Plant Cell Physiol. 48: 843-855.

Norberg PH, Meier H. 1966. Physical and chemical properties of the gelatinous layer in tension wood fibres of aspen (Populus tremula L.). Holzforschung 20: 174-178.

Okuyama T, Yamamoto H, Yoshida M, Hattori Y, Archer R. 1994. Growth stresses in tension wood: role of microfibrils and lignification. Ann. Sci. For. 51: 291-300.

Onaka F. 1949. Studies on compression and tension wood. Wood Res. 1-88.

Patel RN. 1988. Wood anatomy of the dicotyledons indigenous to New Zealand 17. Tiliaceae. N.Z. J. Bot. 26: 337-343.

Pilate G, Chabbert B, Cathala B, Yoshinaga A, Leplé J-C, Laurans F, Lapierre C, Ruel K. 2004a. Lignification and tension wood. C.R. Biol. 327: 889-901.

Pilate G, Déjardin A, Laurans F, Leplé J-C. 2004b. Tension wood as a model for functional genomics of wood formation. New Phytol. 164: 63-72.

Potter MC. 1904. On the occurrence of cellulose in the xylem of woody stems. Ann. Bot. 18: 121-140.

Prodhan AKMA, Funada R, Ohtani J, Abe H \& Fukazawa K. 1995a. Orientation of microfibrils and microtubules in developing tension-wood fibres of Japanese ash (Fraxinus mandshurica var. japonica). Planta. 196: 577-585.

Prodhan AKMA, Ohtani J, Funada R, Abe H, Fukazawa K. 1995b. Ultrastructural investigation of tension wood fibre in Fraxinus mandshurica Rupr. var. japonica Maxim. Ann. Bot. 75: 311-317.

Roussel J-R, Clair B. 2015. Evidence of the late lignification of the G-layer in Simarouba tension wood, to assist understanding how non-G-layer species produce tensile stress. Tree Physiol. 35: 1366-1377.

Ruelle J. 2006. Analyse de la diversité du bois de tension de 3 espèces d'angiospermes de forêt tropicale humide de Guyane Française. Thesis, Université des Antilles et de la Guyane, Cayenne, France. 210 pp.

Ruelle J. 2014. Morphology, anatomy and ultrastructure of reaction wood. In: Gardiner B, Barnett J, Saranpää P, Gril J (eds.), The biology of reaction wood: 13-35. Springer, Berlin, Heidelberg.

Ruelle J, Beauchene J, Thibaut A, Thibaut B. 2007a. Comparison of physical and mechanical properties of tension and opposite wood from ten tropical rainforest trees from different species. Ann. For. Sci. 64: 503-510. 
Ruelle J, Beauchêne J, Yamamoto H, Thibaut B. 2011. Variations in physical and mechanical properties between tension and opposite wood from three tropical rainforest species. Wood Sci. Technol. 45: 339-357.

Ruelle J, Clair B, Beauchêne J, Prévost M-F, Fournier M. 2006. Tension wood and opposite wood in 21 tropical rain forest species. 2. Comparison of some anatomical and ultrastructural criteria. IAWA J. 27: 341-376.

Ruelle J, Yamamoto H, Thibaut B. 2007b. Growth stresses and cellulose structural parameters in tension and normal wood from three tropical rainforest angiosperm species. BioResources 2: $235-251$.

Ruelle J, Yoshida M, Clair B, Thibaut B. 2007c. Peculiar tension wood structure in Laetia procera (Poepp.) Eichl. (Flacourtiaceae). Trees 21: 345-355.

Saiki H, Ono K. 1971. Cell wall organization of gelatinous fibers in tension wood. Bull. Kyoto Univ. For. 42: 210-220.

Sanio KG. 1860a. Einige Bemerkungen über den Bau des Holzes. II. Über die tertiäre Verdickungsschicht des Holzzellen. Bot. Ztg. 23: 201-204.

Sanio KG. 1860b. Einige Bemerkungen über den Bau des Holzes I. Über den Bau des Tüpfels und Hofes. Bot. Ztg. 22: 193-200.

Sanio KG. 1863. Vergleichende Untersuchungen über die Elementarorgane des Holz-Körpers. Bot. Ztg. 21: 101-111.

Schmitt U, Peek R-D, Wong A. 1995. A note on the fine structure of soft rot decay in the polylamellate fibre walls of kempas (Koompassia malaccensis Maing. ex Benth.). Holz Roh- Werkst. 54: 42-42.

Schwarze FWMR, Fink S. 1998. Host and cell type affect the mode of degradation by Meripilus giganteus. New Phytol. 139: 721-731.

Scurfield G. 1972. Histochemistry of reaction wood cell walls in two species of Eucalyptus and in Tristania conferta R.Br. Aust. J. Bot. 20: 9-26.

Scurfield G. 1973. Reaction wood: its structure and function: lignification may generate the force active in restoring the trunks of leaning trees to the vertical. Science 179: 647-655.

Scurfield G, Wardrop AB. 1963. The nature of reaction wood. VII. Lignification in reaction wood. Aust. J. Bot. 11: 107-116.

Singh AP, Wong AHH, Kim YS, Wi SG. 2004. The relationship of fiber cell wall ultrastructure to soft rot decay in kempas (Koompassia malaccensis) heartwood. Ljubljana, Slovenia.

Streit W, Fengel D. 1995. Formation and deposition of tannins in Quebracho colorado (Schinopsis balansae Engl.). Holz Roh- Werkst. 53: 56-60.

Sujan K, Yamamoto H, Matsuo M, Yoshida M, Naito K, Shirai T. 2015. Continuum contraction of tension wood fiber induced by repetitive hygrothermal treatment. Wood Sci. Technol. 49: 1157-1169.

Sultana RS, Ishiguri F, Yokota S, Iizuka K, Hiraiwa T, Yoshizawa N. 2010. Wood anatomy of nine Japanese hardwood species forming reaction wood without gelatinous fibers. IAWA J. 31: 191-202.

Timell TE. 1986. Compression wood in gymnosperms. Springer, Berlin.

Tuskan GA, Difazio SP, Teichmann T. 2004. Poplar genomics is getting popular: the impact of the poplar genome project on tree research. Plant Biol. 6: 2-4.

Wahlgren HE. 1957. Tension wood in overcup oak. U.S. Dept. of Agriculture, Forest Service, Forest Products Laboratory.

Wardrop AB. 1964. The reaction anatomy of arborescent angiosperms. In: Zimmermann MH (ed.), The formation of wood in forest trees: 405-456. Academic Press.

Wardrop AB, Dadswell HE. 1955. The nature of reaction wood. IV. Variation in the cell wall organization of tension wood fibres. Aust. J. Bot. 3: 177-189. 
Yoshida M, Ohta H, Yamamoto H, Okuyama T. 2002. Tensile growth stress and lignin distribution in the cell walls of yellow poplar, Liriodendron tulipifera Linn. Trees 16: 457-464.

Yoshinaga A, Kusumoto H, Laurans F, Pilate G, Takabe K. 2012. Lignification in poplar tension wood lignified cell wall layers. Tree Physiol. 32: 1129-1136.

Yoshizawa N, Inami A, Miyake S, Ishiguri F, Yokota S. 2000. Anatomy and lignin distribution of reaction wood in two Magnolia species. Wood Sci. Technol. 34: 183-196.

Accepted: 4 October 2016

\section{Appendix}

Number of publications mentioning (1) "tension wood" and (2) "tension wood" and poplar or Populus or aspen in the text. The ratio (2): (1) illustrates the increasing focus on poplar in research on tension wood (sources: Web of Science, 04/04/2016).

\begin{tabular}{cccc}
\hline & (1) “tension wood" & $\begin{array}{c}\text { (2) “tension wood" and } \\
\text { poplar or Populus or aspen }\end{array}$ & $\begin{array}{c}\text { Proportion } \\
(2):(1)\end{array}$ \\
\hline $1950-1995$ & 36 & 8 & $22 \%$ \\
$1996-2005$ & 106 & 39 & $37 \%$ \\
$2006-2013$ & 247 & 129 & $52 \%$ \\
$2014-2016$ & 62 & 41 & $66 \%$ \\
\hline
\end{tabular}

